\title{
FACTORIZATION OF THE GREEN'S OPERATOR AND WEAK-TYPE ESTIMATES FOR A RANDOM WALK ON A TREE
}

\author{
Richard Rochberg and Mitchell Taibleson
}

1. Introduction. Let $X$ be a tree, which is to say, a connected graph without loops. The order of a vertex of the tree is the number of edges that meet at the vertex. To avoid some messy, but essentially trivial, complications we will assume throughout that the order of every vertex is at least three. We say that a vertex $u$ is a neighbor of the vertex $v$ if $u$ and $v$ are connected by an edge. When $u$ and $v$ are neighbors we write $u \sim v$. The set of transition probabilities $\{p(u, v)\}$ is said to determine a nearest neighbor random walk if $0 \leq p(u, v)<1$ for all $u, v \in X$ and $p(u, v)>0$ if and only if $u \sim v$. The walk is said to be stochastic if $\sum_{v} p(u, v)=1$ for all $u$ in the tree. The transition probabilities determine a transition operator, $P$, as follows: For $f$ a function defined on the (vertices of the) tree,

$$
P f(u)=\sum_{v \in X} p(u, v) f(v)
$$

We often identify the transition operator with its associated set of transition probabilities.

We assume further that the transition operator $P$ is regular in the sense that there is a positive number $\delta$ such that $p(u, v) \geq \delta$ whenever $u \sim v$. This implies that the orders of the vertices are bounded above.

The visiting probability, $U(u, v)$, for $u$ and $v$ in the tree is the probability that a walk starting at $u$ will visit $v$. There are two common conventions if $u=v$. The standard convention is that $U(u, u)$ is the probability that the walk will visit $u$ at some time in the future, and this is our definition. The other convention is that $U(u, u)=1$. With this in mind we define:

$$
\alpha(u, v)= \begin{cases}U(u, v), & \text { if } u \neq v \\ 1, & \text { if } u=v\end{cases}
$$

$P$ is transient if $U(u, v)<1$ for all $u$ and $v$ in the tree. We will reçuire that $P$ be strongly transient in the sense that there is a $\delta>0$ so that $U(u, v) \leq 1-\delta$ 
whenever $u \sim v$. In the Appendix to [KPT] it is shown that if there is a positive number $\gamma$ such that $p(u, v) \leq 1 / 2-\gamma$ then $P$ is strongly transient.

Let $q+1 \equiv q(u)+1$ be the order of the vertex $u$. We say that the transition operator $P$ is isotropic if $p(u, v)=1 /(q+1)$ whenever $u \sim v$; we say that it is symmetric if $p(u, v)=p(v, u)$ for all $u$ and $v$. A tree is homogeneous if all vertices are of the same order. A transition operator is homogeneous if it is defined on a homogeneous tree and the set of transition probabilities at each vertex are the same. It is easy to see that both an isotropic transition operator on an order bounded tree and a homogeneous symmetric transition operator are always strongly transient. In the sequel we assume that $P$ is strongly transient and we set

$$
\bar{\alpha}=\sup \{\alpha(u, v): u \sim v\} .
$$

Thus, $0<\bar{\alpha}<1$.

Throughout this paper functions on the tree $X$ are complex valued, $X$ is supplied with the discrete topology and the atomic measure which assigns mass one to each vertex. We think of the tree as a collection of vertices and of edges as a relationship on the tree. From this point of view $X$ is a locally compact measure space. For a function, $f$, on the tree,

$$
\begin{aligned}
\|f\|_{p} & =\left(\sum_{u \in X}|f(u)|^{p}\right)^{1 / p}, \quad 0<p<\infty \\
\|f\|_{\infty} & =\sup _{u \in X}|f(u)| .
\end{aligned}
$$

The Laplacian operator, $\Delta$, is defined by

$$
\Delta f(u)=\sum_{v \sim u} p(u, v) f(v)-f(u) .
$$

That is, $\Delta=P-I$. The Green's operator, $G$, speaking loosely, is the inverse of $-\Delta$. It is defined by a kernel $G(u, v)$, the Green's kernel,

$$
G f(u)=\sum_{v \in X} G(u, v) f(v) .
$$

We determine $G$ as follows: Fix $u$ and $v$ in $X$. A path in $X$ connecting $u$ to $v$ is a finite sequence of vertices, $\omega=\left\{\omega_{0}, \omega_{1}, \ldots, \omega_{n}\right\}$, where $\omega_{0}=u, \omega_{n}=v$ and for each $k=1, \ldots, n, \omega_{k-1}$ is a neighbor of $\omega_{k}$. The length of the path $\omega$ is $\ell(\omega)=n$. The weight of the path $\omega$ is

$$
W(\omega)=\prod_{k=1}^{n} p\left(\omega_{k-1}, \omega_{k}\right) .
$$

A trivial path $\omega=\{u\}$ has length zero and weight one. 
Definition. $G(u, v)=\sum_{\omega} W(\omega)$ where $\omega$ ranges over all paths that connect $u$ to $v$.

Observations. It is immediate that $G(u, v)=\sum_{k=0}^{\infty} p^{(k)}(u, v)$ and so $G=\sum_{k=0}^{\infty} P^{k}$ where the $p^{(k)}$ are transition probabilities associated with the transition operator $P^{k}$. It is not difficult to see that $G(u, v)$ is the expected number of visits to $v$ of a random walk that starts at $u$. We note further that $P$ is transient if and only if $G(u, v)<\infty$ for all $u$ and $v$. We do not use any of these observations.

For any two vertices, $u$ and $v$, in $X$ there is a unique path of shortest length that connects $u$ to $v$. This path is called the geodesic that connects $u$ to $v$. We denote its length as $d(u, v)$, and observe that $d$ is a metric on $X$.

General references for matters raised in this introduction are [C], [KPT], and $[G]$.

2. Disk realization of the tree. There are two natural ways to give an orientation to the edges in a tree, the disk realization of the tree and the half-plane realization, which we describe in the next section.

For the disk picture, an arbitrary vertex is selected and is denoted o. It is viewed as the initial point of a random walk that is governed by the transition probabilities $\{p(u, v)\}$. A point on the boundary of $X, \partial X$, is a semi-infinite geodesic, $x=\left\{x_{0}, x_{1} \ldots, x_{k}, \ldots\right\}$, where $x_{0}=o$. Note that $d\left(\mathrm{o}, x_{k}\right)=k$ for all $k$. Suppose $u$ and $v$ are in $X$. We say that $w$ is between $u$ and $v$ if $w$ is on the geodesic that connects $u$ to $v$. If $x \in \partial X$ then $w$ is between $x$ and $u$ if $w$ is between $u$ and $x_{k}$ for all $k$ large enough. Let $D=X \cup \partial X$. If $v$ is between $o$ and $u$ we say that $v$ is above $u$, or that $u$ is below $v$. If $x=\left\{x_{0}, x_{1} \ldots, x_{k}, \ldots\right\}$ we say that the geodesic $\left\{x_{0}, x_{1} \ldots, x_{k}, \ldots\right\}$ connects $x$ to $o$. It follows that $o$ is above every point in $D$ and if $x \in \partial X$ then $x$ is below $x_{k}$ for all $k$.

For each vertex $u$ there are $q(u)$ neighbors of $u,\left\{u_{j}\right\}$, that are below $u$, except for o that has $q(0)+1$ lower neighbors. For each vertex $u, u \neq 0$, there is a unique vertex $u^{-}$such that $u^{-} \sim u$ and $u^{-}$is above $u$.

We now define a subbase for the topology of $D$. It consists of all sets $N(u)$, $u \in X$ where $N(u)=\{v: v$ is below $u\}$. With this topology $D$ is compact, the restriction of the topology to $X$ is discrete, and its restriction to $\partial X$ is compact. If $\left\{x_{0}, x_{1} \ldots, x_{k}, \ldots\right\} \in \partial X$ then $N\left(x_{k}\right) \cap \partial X$ is referred to as an interval of level $k$. We say that $x_{k}$ is a vertex of level $k$. We write $I_{u}=N(u) \cap \partial X$.

We now define the hitting (harmonic) measure, $\mu$, on $\partial X$. Denote by $F_{n}$ the (random) vertex at step $n$ for the random walk determined by $P$ with $F_{0}=0$.

$$
\mu\left(I_{u}\right)=\operatorname{Pr}\left(\exists k_{0}: F_{k} \in N(u) \quad \forall k \geq k_{0} \mid F_{0}=\mathrm{o}\right)
$$

$\mu$ extends to a Borel measure on $\partial X$.

The function $U(u, v)$ defined in Section 1, called the visiting probability, is formally defined by:

$$
U(u, v)=\operatorname{Pr}\left(3 n>0: F_{n}=v \mid F_{0}=u\right) .
$$


Consider the random walk determined by $P$ starting at $u$ and conditioned to remain in $N(u)$ from some step onward. Define a hitting measure, $\nu$, for this walk just as we defined $\mu$. Then $\nu\left(I_{u}\right)=1$ and for $v \sim u, v \neq u^{-}$, define the relative forward probability, $\pi(u, v)=\nu\left(I_{v}\right)$. Clearly

$$
\sum_{\nu: v^{-}=u} \pi(u, v)=\nu\left(I_{u}\right)=1
$$

We say that we are moving forward if we move to a position below.

By Proposition 2 of [KPT], for $u \neq 0$

$$
\pi\left(u^{-}, u\right)=\frac{p\left(u^{-}, u\right)\left(1-U\left(u, u^{-}\right)\right)}{\sum_{w: w^{-}=u^{-}} p\left(u^{-}, w\right)\left(1-U\left(w, u^{-}\right)\right)}
$$

Observe that this formula shows that the probability of being in $N(u)$ from some step onward, conditioning on the event of being in $N\left(u^{-}\right)$from some step onward, is the same as that of the event of starting at $u^{-}$, conditioning on the event of never returning to $u^{-}$and being in $N(u)$ from the first step onward. Let $A(u)$ be the probability that a random walk starting at $u$ moves forward on its first step and never returns to $u$. We see that $A\left(u^{-}\right)$is the denominator in equation (1), so that

$$
\pi(u, v)=\frac{p(u, v)(1-U(v, u))}{A(u)}, \quad \text { if } u=v^{-} .
$$

Notation. Suppose $u$ is a vertex and $u \neq 0$. Let $u_{j}, j=1, \ldots, q$ be the neighbors of $u$ that are below $u$. We set $u^{-}=u_{0}, p_{j}=p\left(u, u_{j}\right), j=0,1, \ldots, q$; $\pi_{j}=\pi\left(u, u_{j}\right), \alpha_{j}=\alpha\left(u_{j}, u\right), j=1, \ldots, q ;$ and $\alpha_{0}=\alpha\left(u, u_{0}\right)$.

Lemma 1. $A(\mathrm{o})=1-U(\mathrm{o}, \mathrm{o})$. If $u \neq$ o then

$$
A(u)=p\left(u, u^{-}\right)\left(1-U\left(u, u^{-}\right)\right) / U\left(u, u^{-}\right) .
$$

Proof: The result for $A(0)$ follows from the definitions. For $u \neq 0$ we use the relationship

$$
\alpha_{0}=p_{0}+\sum_{j=1}^{q} p_{j} \alpha_{j} \alpha_{0},
$$

which follows from the Markov property for randon walks (see [KPT]). We rewrite this

$$
\sum_{j=1}^{q} p_{j} \alpha_{j}=1-\frac{p_{0}}{\alpha_{0}}=\sum_{j=1}^{q} p_{j}+p_{0}\left(1-\frac{1}{\alpha_{0}}\right)
$$


From this we obtain

$$
\sum_{j=1}^{q} p_{j}\left(1-\alpha_{j}\right)=\frac{1-\alpha_{0}}{\alpha_{0}} \cdot p_{0}
$$

Using the notation introduced before the statement of Lemma I we note that

$$
A(u)=\frac{p_{0}\left(1-\alpha_{0}\right)}{\alpha_{0}}, \quad \pi_{j}=\frac{p_{j}\left(1-\alpha_{j}\right)}{p_{0}\left(1-\alpha_{0}\right)} \alpha_{0},
$$

when $u \neq 0$. It is easy to see that if $\left\{\omega_{0}, \omega_{1}, \ldots, \omega_{n}\right\}$ is the geodesic connecting o to $u$ then

$$
\mu\left(I_{u}\right)=\prod_{k=1}^{n} \pi\left(\omega_{k-1}, \omega_{k}\right)
$$

If $\omega=\left\{\omega_{0}, \omega_{1}, \ldots, \omega_{n}\right\}$ is the geodesic connecting $u$ to $v$ we define

$$
V(u, v)=W(\omega)
$$

and observe that

$$
\alpha(u, v)=\prod_{k=1}^{n} \alpha\left(\omega_{k-1}, \omega_{k}\right)
$$

Observation. It is clear that the regularity and strong transitivity of the waik imply that there is a $\bar{\delta}>0$ such that whenever $u$ and $v$ are vertices and $u=v^{-}$then $\pi(u, v) \geq \bar{\delta}$. and $A(u) \sim 1$ for all vertices $u$.

Theorem 2. If $u$ and $v$ are vertices and $u$ is above $v$ then

$$
\frac{\mu\left(I_{v}\right)}{\mu\left(I_{u}\right)}=\frac{V(u, v)}{V(v, u)} \frac{A(v)}{A(u)} U(v, u)
$$

Proof: Use equations (2)-(8), gather terms and simplify.

3. Half-plane realization of the tree. We begin with the disk picture. Select a point on $\partial X$ and denote it by $\infty$. To each other point, $y$ on $\partial X$ we associate the unique doubly infinite geodesic $\left\{\ldots, y_{-2}, y_{-1}, y_{0}, y_{1}, y_{2}, \ldots\right\}$ such that $\left\{y_{0}, y_{1}, \ldots\right\}$ is cofinal with the geodesic in $D$ that defines $y$ and $\left\{y_{0}, y_{-t}, \ldots\right\}$ is cofinal with the geodesic defining $\infty$. For this realization we call the tree $Y$ and the finite part of the boundary is $\partial Y, H=Y \cup \partial Y$. For each vertex $u$ there is a unique geodesic that connects $u$ to $\infty$; which is to say a half-infinite geodesic with initial point $u$ that is cofinal with the geodesic in 
$X$ that defines $\infty$. We say that each vertex on that geodesic is above $u$, and that $v$ is below $w$ whenever $w$ is above $v$. The point $\infty$ is above every other point in $H$, and a point on $\partial Y$ is below every vertex that lies on the geodesic that connects it to $\infty$. A crucial (simplifying) difference with the disk picture is that for every vertex, $u$, on the tree there is a unique vertex, $u^{-}$, that is a neighbor of $u$ and is above $u$.

As before we set $N(u)=\{v \in H: v$ is below $u\}$. This defines a subbase for a topology on $H$ that is locally compact. The restriction of the topology to $Y$ is discrete and its restriction to $\partial Y$ is locally compact. Again we set $I_{u}=N(u) \cap \partial Y$. Just as each vertex in the disk has a level we can define a Jevel for each vertex in the half-plane. Select a reference vertex $e$, and consider the geodesic $\left\{y_{0}, y_{-1}, y_{-2}, \ldots\right\}$ that connects $\mathbf{e}$ to $\infty$. Then we set the level of $\mathbf{e}$ as 0 and each vertex, $u$, on the geodesic has level $-d(\mathbf{e}, u)$. Observe that every vertex on the tree is below some vertex on that half-infinite geodesic. Using the rule: the level of $u$ is the level of $u^{-}$plus one, a level for each vertex is defined.

As in Section 2 we can define the functions: $A(u)$ and $V(u, v)$. To define $A(u)$ we no longer need a special case. For all vertices u

$$
A(u)=p\left(u, u^{-}\right)\left(1-\alpha\left(u, u^{-}\right)\right) / \alpha\left(u, u^{-}\right) .
$$

Furthermore, we may define the rclative forward probabilities

$$
\pi(u, v)=p(u, v)(1-\alpha(v, u)) / A(u)
$$

whenever $u=v^{-}$.

Since the boundary is not compact we may no longer define the $\mu\left(I_{u}\right)$ as probabilities, but we will be able to use conditional probabilities. We proceed by choosing a reference vertex, $v_{0}$. For example, one may choose $v_{0}=\mathbf{e}$. Set $\mu\left(I_{v_{0}}\right)=1$. Extend $\mu$ to the tree by the rule: $\mu\left(I_{u}\right)=\mu\left(I_{u^{-}}\right) \pi\left(u, u^{-}\right) . \quad \mu$ extends to a Borel measure on $\partial Y$. As in Section 3:

Theorem 3. If the vertex $v$ is below the vertex $u$ then

$$
\frac{\mu\left(I_{v}\right)}{\mu\left(I_{u}\right)}=\frac{V(u, v)}{V(v, u)} \frac{A(v)}{A(u)} U(v, u)
$$

Observation. Theorem 2 and Theorem 3 have the same form but the values of the function $A$ are defined differently in the two realizations.

We note that $\mu\left(I_{v}\right) / \mu\left(I_{u}\right)$ is the probability that a random walk that is conditioned to eventually stay in $N(u)$, is eventually in $N(v)$. More informally: it is the probability that the random walk hits the boundary in $I_{v}$ given that it will hit the boundary in $I_{u}$.

We also note that $A(u) \sim 1$. 
4. Factorization of the Laplacian and the Green's operator. We begin with the half-plane; the extension to the disk will be given in Section 6 . below. Recall that every vertex is both above and below itself. If we need to rule out this possibility we will use the locution strictly below or stricly above.

We define the kernels for operators $S$ and $T$.

$$
\begin{gathered}
K_{S}(u, v)= \begin{cases}\frac{\mu\left(I_{v}\right)}{\mu\left(I_{u}\right)}, & \text { if } v \text { is below } u \\
0, & \text { otherwise. }\end{cases} \\
K_{T}(u, v)= \begin{cases}\alpha(u, v)-\alpha\left(u, v^{-}\right), & \text {if } v \text { is above } u \\
0, & \text { otherwise. }\end{cases}
\end{gathered}
$$

Observe that $K_{S}(u, u)=1$ and $K_{T}(u, u)=1-\alpha\left(u, u^{-}\right)$for all vertices $u$. We let,

$$
\begin{aligned}
& S f(u)=\sum_{v} K_{S}(u, v) f(v), \\
& T f(u)=\sum_{v} K_{T}(u, v) f(v),
\end{aligned}
$$

whenever the series converges absolutely.

For the next definitions and the theorem that follows we use the notation introduced just before Lemma $I$ and the formula for $A(u)$ in equation (5). We introduce two difference operators. For $g$ a function defined on the tree:

$$
\begin{gathered}
\Delta^{+} g(u)=\sum_{j=1}^{q} \pi\left(u, u_{j}\right) g\left(u_{j}\right)-g(u) \\
\Delta^{-} g(u)=\frac{1}{1-\alpha\left(u, 2 t^{-}\right)} g(u)-\frac{\alpha\left(u, u^{-}\right)}{1-\alpha\left(u, u^{-}\right)} g\left(u^{-}\right)
\end{gathered}
$$

Theorem 4. For any function $g$ defined on the tree

$$
\Delta^{+} \Delta^{-} g(u)=\frac{1}{A(u)} \Delta g(u)
$$

Proof:

$$
\begin{aligned}
\Delta^{+} \Delta^{-} g(u)= & \sum_{j=1}^{q} \pi_{j} \Delta^{-} g\left(u_{j}\right)-\Delta^{-} g(u) \\
= & \sum_{j=1}^{q} \frac{p_{j}\left(1-\alpha_{j}\right)}{p_{0}\left(1-\alpha_{0}\right)} \alpha_{0}\left\{\frac{1}{1-\alpha_{j}} g\left(u_{j}\right)-\frac{\alpha_{j}}{1-\alpha_{j}} g(u)\right\} \\
& -\left\{\frac{1}{1-\alpha_{0}} g(u)-\frac{\alpha_{0}}{1-\alpha_{0}} g\left(u_{0}\right)\right\}
\end{aligned}
$$




$$
\begin{aligned}
& =\frac{\alpha_{0}}{p_{0}\left(1-\alpha_{0}\right)} \sum_{j=1}^{q} p_{j} g\left(u_{j}\right)-\left[\sum_{j=1}^{q} p_{j} \alpha_{j} \alpha_{0}\right] \frac{1}{p_{0}\left(1-\alpha_{0}\right)} g(u) \\
& -\frac{1}{1-\alpha_{0}} g(u)+\frac{\alpha_{0}}{1-\alpha_{0}} g\left(u_{0}\right) \\
& =\frac{\alpha_{0}}{p_{0}\left(1-\alpha_{0}\right)} \sum_{j=1}^{q} p_{j} g\left(u_{j}\right)+\frac{\alpha_{0}}{p_{0}\left(1-\alpha_{0}\right)} p_{0} g\left(u_{0}\right) \\
& -\left(\frac{\alpha_{0}-p_{0}}{p_{0}\left(1-\alpha_{0}\right)}+\frac{1}{1-\alpha_{0}}\right) g(u) \\
& =\frac{\alpha_{0}}{p_{0}\left(1-\alpha_{0}\right)}\left[\sum_{j=0}^{q} p_{j} g\left(u_{j}\right)-g(u)\right] \\
& =\frac{ \pm}{A(u)} \Delta g(u) \text {. }
\end{aligned}
$$

At the fourth step we used equation (4). This completes the proof.

We show next that $\Delta^{-}$is the inverse of $T$ and that $-\Delta^{+}$is the inverse of $S$ on appropriate domains. Suppose $f$ is in the domain of $T$. It is easy to see that

$$
T f(u)=\left(1-\alpha\left(u, u^{-}\right)\right) f(u)+\alpha\left(u, u^{-}\right) T f\left(u^{-}\right)
$$

Solving for $f$ we obtain

$$
\begin{aligned}
f(u) & =\frac{1}{1-\alpha\left(u, u^{-}\right)} T f(u)-\frac{\alpha\left(u, u^{-}\right)}{1-\alpha\left(u, u^{-}\right)} T f\left(u^{-}\right) \\
& =\Delta^{-} T f(u) .
\end{aligned}
$$

Since the coefficients that define $T f(u)$ are non-negative and sum to one, $\ell^{\infty}$ (and so $\ell^{p}, 0<p \leq \infty$ ) is in the domain of $T$. Thus on $\ell^{p}, 0<p \leq \infty$, $\Delta^{-} T=I$.

There is an $\bar{\alpha}$ such that $0<\alpha\left(u, u^{-}\right) \leq \bar{\alpha}<1$ for all $u$. Consequently if $v$ is above $u, \alpha(u, v) \leq \ddot{\alpha}^{d(u, v)}$. Consider the semi-infinite geodesic $\left\{x_{0}, x_{1}, x_{2}, \ldots\right\}$, 
where $x_{0}=u$, and $x_{k+1}=x_{k}^{-}$for $k=1,2, \ldots$ Then

$$
\begin{aligned}
T \Delta^{-} f(u)= & \sum_{k=0}^{\infty}\left(\alpha\left(x_{0}, x_{k}\right)-\alpha\left(x_{0}, x_{k+1}\right)\right) \Delta^{-} f\left(x_{k}\right) \\
= & \sum_{k=0}^{\infty} \alpha\left(x_{0}, x_{k}\right)\left(1-\alpha\left(x_{k}, x_{k+1}\right)\right) \times \\
& \quad \times\left[\frac{1}{I-\alpha\left(x_{k}, x_{k+1}\right)} f\left(x_{k}\right)-\frac{\alpha\left(x_{k}, x_{k+1}\right)}{1-\alpha\left(x_{k}, x_{k+1}\right)} f\left(x_{k+1}\right)\right] \\
= & \lim _{N \rightarrow \infty} \sum_{k=0}^{N} \alpha\left(x_{0}, x_{k}\right) f\left(x_{k}\right)-\alpha\left(x_{0}, x_{k+1}\right) f\left(x_{k+1}\right) \\
= & f\left(x_{0}\right)-\lim _{N \rightarrow \infty} \alpha\left(x_{0}, x_{N+1}\right) f\left(x_{N}+1\right) \\
= & f(u)
\end{aligned}
$$

provided $\alpha\left(x_{0}, x_{k+1}\right) f\left(x_{k+1}\right)=\alpha(1)$ as $k \rightarrow \infty$. This certainly holds if $f$ is bounded and so:

Theorem 5. $\Delta^{-}$is the inverse of $T$ on $\ell^{p}, 0<p \leq \infty$.

Lemma 6. $T$ is bounded on $\ell^{\infty}$ with norm $1 . T$ and $\Delta^{-}$map $\ell^{\infty}$ onto itself.

Proof: It is easy to see that $T$ is bounded on $\ell^{\infty}$ with norm $I$ and it is trivial that $\Delta^{-}$is bounded on $\ell^{\infty}$ with norm at most $(1+\bar{\alpha}) /(1-\bar{\alpha})$. It follows that $T$ and $\Delta^{-} \operatorname{map} \ell^{\infty}$ onto $\ell^{\infty}$.

We now consider $S$ and $\Delta^{+}$. Note that $S$ is not defined on $\ell^{\infty}$. In fact if $f(u) \equiv 1$ then $S f(u) \equiv \infty$. To see this let

$$
\left\{x_{k j}\right\}, \quad j=\mathrm{I}, \ldots, j(k) ; \quad k=0,1,2, \ldots,
$$

be the $j(k)$ vertices that are below $u$ at distance $k$. Then

$$
S f(u)=\sum_{k=0}^{\infty} \sum_{j=1}^{j(k)} \frac{\mu\left(I_{x_{k j}}\right)}{\mu\left(I_{u}\right)} \cdot 1=\sum_{k=0}^{\infty} 1=\infty .
$$

Observation. There is a $\bar{\delta}, 0<\bar{\delta}<1$ such that $\pi\left(u^{-}, u\right) \leq 1-\bar{\delta}$ for all $u$. This follows from the regularity and strong transience of $P$.

Lemma 7. If $f$ is in $\ell^{p}, 0<p<\infty$, then $f$ is in the domain of $S$.

Proof: Suppose first that $1 \leq p<\infty$.

$$
\begin{aligned}
\left|\sum_{j=1}^{j(k)} \frac{\mu\left(I_{x_{k}}\right)}{\mu\left(I_{u}\right)} f\left(x_{k j}\right)\right| & \leq\left(\sum_{j=1}^{j(k)}\left|f\left(x_{k j}\right)\right|^{p} \frac{\mu\left(I_{x_{k j}}\right)}{\mu\left(I_{u}\right)}\right)^{1 / p} \\
& \leq(1-\bar{\delta})^{k / p}\|f\|_{p}
\end{aligned}
$$


Thus,

$$
|S f(u)| \leq\left(\sum_{k=0}^{\infty}(1-\bar{\delta})^{k / p}\right)\|f\|_{p}=\frac{1}{1-(1-\bar{\delta})^{1 / p}}\|f\|_{p} .
$$

When $0<p<1, \ell^{p} \subset \ell^{1}$, and so for all $p, 0<p<\infty$, $\ell^{p}$ is in the domain of $S$.

For $f$ in the domain of $S$ we see that

$$
S f(u)=f(u)+\sum_{j=1}^{q(u)} \pi\left(u, u_{j}\right) S f\left(u_{j}\right)
$$

where the $u_{j}$ are the lower neighbors of $u$. That is,

$$
-\Delta^{+} S f(u)=f(u)
$$

for all $f \in \ell^{p}, 0<p<\infty$. For such an $f$ we have, using equation (13),

$$
\lim _{k \rightarrow \infty} \sum_{j=1}^{j(k)} \frac{\mu\left(I_{x_{k j}}\right)}{\mu\left(I_{u}\right)} f\left(x_{k j}\right)=0 .
$$

Let $\left\{x_{k j l}\right\}$ be the $q\left(x_{k j}\right)$ lower neighbors of $x_{k j}$.

$$
\begin{aligned}
S \Delta^{+} f(u) & =\sum_{k=0}^{\infty} \sum_{j=1}^{j(k)} \frac{\mu\left(I_{x_{k j}}\right)}{\mu\left(I_{u}\right)}\left\{\sum_{i=1}^{q\left(x_{k j}\right)} \pi\left(x_{k j}, x_{k j l}\right) f\left(x_{k j l}\right)-f\left(x_{k j}\right)\right\} \\
& =\lim _{K \rightarrow \infty} \sum_{k=0}^{K}\left[\sum_{j=1}^{j(k+1)} \frac{\mu\left(I_{x_{k+1, j}}\right)}{\mu\left(I_{u}\right)} f\left(x_{k+1, j}\right)-\sum_{j=1}^{j(k)} \frac{\mu\left(I_{x_{j}}\right)}{\mu\left(I_{u}\right)} f\left(x_{k j}\right)\right] \\
& =\lim _{K \rightarrow \infty} \sum_{j=1}^{j(K+1)} \frac{\mu\left(I_{x_{K+1, j}}\right)}{\mu\left(I_{u}\right)} f\left(x_{K+1, j}\right)-f(u) .
\end{aligned}
$$

Theorem 8. $-\Delta^{+}$is the inverse of $S$ on $\ell^{p}, 0<p<\infty$.

Lerruma 9. S is lowanded on $\ell^{4} . S$ and $\Delta^{+}$map $\ell^{1}$ onto itself.

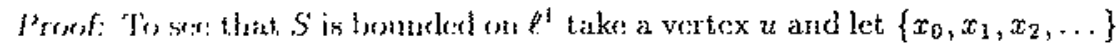

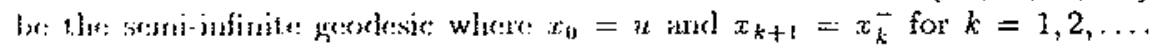
The:n

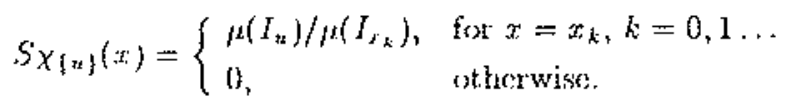


Thus,

$$
\begin{aligned}
\left\|S_{\chi\{u\}}\right\|_{1} & =\sum_{k=0}^{\infty} \frac{\mu\left(I_{u}\right)}{\mu\left(I_{x_{k}}\right)} \\
& \leq \sum_{k=0}^{\infty}(1-\bar{\delta})^{k}=\frac{1}{\bar{\delta}}
\end{aligned}
$$

But we can write

$$
\begin{aligned}
f & =\sum f(v) \chi\{v\} \\
S f & =\sum f(v) S_{\chi\{v\}} .
\end{aligned}
$$

It follows that

$$
\|S f\|_{1} \leq \sum|f(v)|\left\|S \chi_{\{v\}}\right\|_{1} \leq\|f\|_{1} / \bar{\delta}
$$

We now define the multiplier transform $A$ and its inverse $R$.

$$
A f(u)=A(u) f(u) \quad R f(u)=\frac{1}{A(u)} f(u)
$$

It will be convenient to set $R(u)=1 / A(u)$.

Proposition 10. The operators $A$ and $R$ are bounded on $l^{p}, 0<p \leq \infty$.

Proof: This is inmediate from the fact that $A(u) \sim 1$.

The theorems of this section suggest that $G=T S R$.

Theorem 11.

$$
G(u, v) A(v)=K_{T S}(u, v)=\sum_{w} K_{T}(u, w) K_{S}(w, v)
$$

for all vertices $u$ and $v$ on the tree.

Proof: $G(u, v)$ is the sum, over all paths from $u$ to $v$, of the probabilities of traveling such paths. $A(v)$ is the probability of the event of moving from $v$ towards the boundary and never returning to $v$. So $G(u, v) A(v)$ is the sum of the probabilities for traveling certain paths, where the sum is over all paths that start from $u$ go to $v$ and then move toward the boundary, never to return to $v$. 
The kernel for $T S$ is

$$
K_{T S}(u, v)=\sum_{w} K_{T}(u, w) K_{S}(w, v)
$$

where $v$ ranges over all vertices that are above both $u$ and $v$, since otherwise the summand is zero. Let $w_{0}$ be the lowest such point and let $w_{k+1}=w_{k}{ }^{-}$, $k=0,1,2, \ldots$ Then

$$
\begin{aligned}
K_{T S}(u, v) & =\sum_{k=0}^{\infty} K_{T}\left(u, w_{k}\right) K_{S}\left(w_{k}, v\right) \\
& =\sum_{k=0}^{\infty}\left(\alpha\left(u, w_{k}\right)-\alpha\left(u, w_{k+1}\right)\right) \frac{\mu\left(I_{v}\right)}{\mu\left(I_{w_{k}}\right)}
\end{aligned}
$$

Each path from $u$ to $v$ will pass through $w_{0}$ and will continue to some $w_{k}$, but not to $w_{k+1}, k=0,1,2, \ldots$. The $k^{\text {th }}$ term of the expansion (14) involves just those paths and an examination of the definition of $\mu$ shows that $\mu\left(I_{v}\right) / \mu\left(I_{w_{k}}\right)$ is precisely the probability that a random walk starting at $w_{k}$ will pass through $v$ on its way to the boundary never to return to $v$. Since a path goes to the boundary with probability one this establishes the equality.

Remarks. The crucial fact in this development is the factorization of the Laplacian which is presented in Theorem 4 without any motivation. This result is a reformulation of results presented in [KPT]. In that paper a correspondence is established between harmonic functions on the tree; that is functions $F$ such that $\Delta F=0$, and functions $f$ that are boundary martingales associated with $P$; which in the language of this paper means that $\Delta^{+} f=0$. The correspondence is established by the relation $F=T f$.

Corollary 12. $G=T S R$ on $\ell^{p}, 0<p<\infty$.

5. Boundedness results. If $E$ is a finite subset of the tree let $|E|$ denote the cardinality of the set $E$.

Lemma 13. Suppose that $E$ is a finite subset of the tree. Then there is a set $F, F \subset E,|F|>(1 / 2)|E|$, such that $S_{\chi_{F}(x)} \leq C$ where $C$ is a positive constant that is independent of $E$.

Proof: We foliow, as a model, the proof of Proposition 3 in [RT]. We say that $v$ is a descendant of $u$ if $v$ is strictly below $u$, and that $v$ is a descendant of $u$ that derives from a lower neighbor $u_{j}$ of $u$ if $v=u_{j}$ or is a descendant of $u_{j}$. For $E$ a finite subset of the tree let $\partial_{L} E$ be the lower boundary of $E$ which we define as the subset of vertices in $E$ that do not have descendants from each of its lower neighbors. 
Assume for now that there is a vertex, $x$, in $E$ such that every vertex in $E$ is below $x$. In this case we say that $E$ is triangular. Let $Z$ be the subtree of $Y$ that contains $x$ and all of its descendants. An end of $E$ is a vertex in $E$ that has no descendents in $E$. End is the set of ends of $E$. A vertex in $Z$ is a branch point of $E$ if it has at least two descendants in $E$. Brch is the set of branch points. Since $q(u) \geq 2$ the number of ends of $E$ is greater than or equal to one plus the number of branch points. Since every end is in the lower boundary

$$
\left|\partial_{L} E\right| \geq \mid \text { End }|\geq| \text { Brch } \mid+1 \text {. }
$$

An interior point of $E$ is a point in $E$ that is not in the lower boundary. Int is the set of interior points of $E$. An interior point has descendants from all of its lower neighbors and so it is a branch point. Thus,

$$
|\operatorname{Int}| \leq|\operatorname{Brch} \cap E| \leq|\operatorname{Brch}|
$$

Notice that

$$
|E|=\left|\partial_{L} E\right|+|\operatorname{Int}|
$$

It follows from (15) and (16) that

$$
\left|\partial_{L} E\right|>|\operatorname{Brch}| \geq|\operatorname{Int}|,
$$

and so $\left|\partial_{L} E\right|>\frac{1}{2}|E|$. Since $E$ can be written as a finite union of pairwise disjoint triangular sets in such a way that the lower boundary of $E$ is the union of the lower boundaries of the pieces we see that $\left|\partial_{L} E\right|>\frac{1}{2}|E|$ for any finite set. (The first piece is a highest point in $E$ and all of its descendants in $E$. Then take from the remaining points a highest point and all of its descendants. Continue in this way until all points in $E$ are exhausted.)

Let $F$ be the lower boundary of $E$. In order to estimate $S \chi_{F}(x)$ we first observe that for all vertices with a level below the lowest level of a vertex in $E$ we have $S \chi_{F}(x)=0$, and for vertices on the same level as the lowest vertex (or vertices) of $E$ we have that $S_{\chi F}(x)=1$ or 0 depending on whether or not $x \in F$. Recall (see the last line of 3 ) that $\pi\left(u^{-}, u\right) \geq \vec{\delta}>0$ for all vertices $u$.

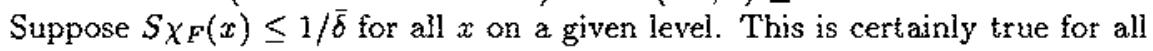
vertices at or below the lowest level of a vertex in $E$. Take a vertex $x$ on the next higher level.

$$
S_{\chi F}(x)=\chi_{F}(x)+\sum_{j=1}^{q} \pi_{j} S_{\chi_{F}}\left(x_{j}\right),
$$

where the $x_{j}$ are the $q$ lower neighbors of $x$ and $\pi_{j}=\pi\left(x, x_{j}\right)$. There are two cases to consider. If $x \notin F$

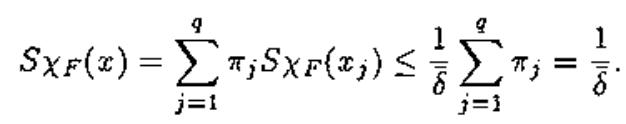


If $x \in F$ then at least one $x_{j_{0}}$ has no descendant in $F$ and $S \chi_{F}\left(x_{j_{0}}\right)=0$. Thus,

$$
S_{\chi F}(x)=1+\sum_{j \neq j_{0}} \pi_{j} S_{\chi F}\left(x_{j}\right) \leq 1+\left(1-\pi_{j_{0}}\right) \frac{1}{\bar{\delta}} \leq 1+\frac{1-\bar{\delta}}{\bar{\delta}}=\frac{1}{\bar{\delta}} .
$$

This completes the proof.

We now define the formal adjoints of $R, S$, and $T . R^{*}=R, S^{*}$ and $T^{*}$ are defined as the operators with kernels:

$$
K_{S^{*}}(u, v)=K_{S}(v, u), \quad K_{T}(u, v)=K_{T}(v, u) .
$$

Theorem 14. $S^{*}$ is of weak type $(1,1)$. That is, suppose that $f \in \ell^{1}$ and $s \neq 0$. Then

$$
\left|\left\{x:\left|S^{*} f(x)\right|>s\right\}\right| \leq C \frac{1}{s}\|f\|_{1},
$$

Where $C$ is a positive constant that does not depend on $f$ or $s$.

Proof: Clearly we may assume that $f(x) \geq 0$ for all $x$ and that $\|f\|_{1}=1$. Since $f \in \ell^{1}$ we see that $E=\left\{x: S^{*} f(x)>s\right\}$ is finite. Choose the set $F$ as in Lemma 13.

$$
s \frac{1}{2}|E|<\int S^{*} f \cdot \chi_{F}=\int f \cdot S_{\chi F}<\frac{1}{\delta} .
$$

This completes the proof.

Lemma 15. $T^{*}$ is bounded on $\ell^{1}$ with norm $1 . S^{*}$ is bounded on $\ell^{\infty}$.

Proof: Immediate from Lemmas 6 and 9 .

Lemma 16. $S^{*}$ is bounded on $\ell^{p}, 1<p \leq \infty$. $S$ is bounded on $\ell^{p}, 1 \leq p<$ $\infty$.

Proof: By Theorem $14 S^{*}$ is of weak-type $(1,1)$, and by Lemma 15 it is bounded on $\ell^{\infty}$; an application of the Marcinkiewicz Interpolation Theorem completes the argument for $S^{*}$. The result for $S$ follows by duality.

Theorem 17. $S$ maps $\ell^{p}$ onto $\ell^{p}$ when $1 \leq p<\infty$.

Proof: $S$ and its inverse $-\Delta^{+}$are both bounded on $\ell^{p}$.

Observation. $G$ is bounded on $\ell^{p}, 1<p<\infty$ if and only if $T$ is bounded on $\ell^{p}$. This follows from $G=T S R$ and $T=-G A \Delta^{+}$. 
Theorem 18. $G^{*}$ is of weak type $(1,1)$.

Proof: $G^{*}=R S^{*} T^{*}$. We use Theorem 14, Lemma 15, and the fact that $R$ is a bounded function. Suppose $f \in \ell^{1}$ and $s>0$.

$$
\begin{aligned}
\left|\left\{u:\left|G^{*} f(u)\right|>s\right\}\right| & \leq \mid\left\{u: S^{*} T^{*} f(u)>\frac{s}{\|R\|_{\infty}}\right\} \\
& \leq \frac{2}{\bar{\delta}}\left\|T^{*} f\right\|_{1} \frac{\|R\|_{\infty}}{s} \\
& \leq \frac{2}{\bar{\delta}}\|R\|_{\infty}\|f\|_{1} \frac{1}{s}
\end{aligned}
$$

Theorem 19. There is a $p_{0}$, finite and positive, such that $G$ is bounded on $\ell^{p}, p_{0}<p \leq \infty$.

Proof: From our remark above we only need to prove our result for $T$. Notice that the case $p=\infty$ is Lemma 6 , so we may assume that $p$ is positive and finite. Our proof will use Schur's Lemma (see [RW; Prop. 3.50]).

Let $\phi(u)=\bar{\alpha}^{k \epsilon}$ where $k$ is the level of $u$ and $\epsilon$ is a positive number that is chosen below. Observe that $K_{T}(u, v) \leq \bar{\alpha}^{d(u, v)}$ if $u$ is above $v$ and is zero otherwise. Choose $r$ such that $\frac{1}{p}+\frac{1}{r}=1$. We want to show:

$$
\begin{aligned}
& \sum_{v} K_{T}(u, v) \phi^{r}(v) \leq C \phi^{r}(u) \\
& \sum_{u} K_{T}(u, v) \phi^{p}(u) \leq C \phi^{p}(v)
\end{aligned}
$$

for some constant $C$.

Suppose the level of $u$ is $k_{0}$. Then

$$
\begin{aligned}
\sum_{v} K_{T}(u, v) \phi^{r}(v) & \leq \sum_{k=0}^{\infty} \bar{\alpha}^{k} \bar{\alpha}^{\left(k_{0}-k\right) \epsilon r} \\
& =\bar{\alpha}^{k_{0} e r} \sum_{k=0}^{\infty} \bar{\alpha}^{(1-t r) k} \\
& =\frac{1}{1-\bar{\alpha}^{1-\epsilon r}} \phi^{r}(u)
\end{aligned}
$$

provided $0<\epsilon<1 / r$.

Notation. Let $\bar{q}+1$ be the maximum order of a vertex in the tree. 
Suppose $v$ is of level $k_{0}$. Then

$$
\begin{aligned}
\sum_{u} K_{T}(u, v) \phi^{p}(u) & \leq \sum_{k=0}^{\infty} \bar{q}^{k} \bar{\alpha}^{k} \bar{\alpha}^{\epsilon p\left(k_{0}+k\right)} \\
& =\bar{\alpha}^{k_{0} \epsilon p} \sum_{k=0}^{\infty} \bar{\alpha}^{k(1+\epsilon p+\log \bar{q} / \log \bar{\alpha})} \\
& =A_{\epsilon, \bar{\alpha}, \bar{q}} \phi^{p}(v)
\end{aligned}
$$

provided $\epsilon>1 / p(\log \tilde{q} / \log (1 / \bar{\alpha})-1)$. An elementary computation shows that a choice of such an $\epsilon$ is possible provided

$$
p>\frac{\log \bar{q}}{\log (1 / \bar{\alpha})}=p_{1} .
$$

Let $p_{0}=\max \left[1, p_{1}\right]$. Our argument shows that $T$ is bounded on $\ell^{p}$ if $p>p_{0}$.

An example is given in Section 7 for which $p_{0}>1$.

We use a notion introduced by Gerl in [G].

Definition. A nearest neighbor random walk is strongly reversible if there is a function $m(u)$ such that whenever $u$ and $v$ are neighbors $m(u) p(u, v)=$ $m(v) p(v, u)$ and if there are positive constants $m$ and $M$ such that $m(u) p(u, v) \geq$ $m>0$ whenever $u$ and $v$ are neighbors and $m(u) \leq M<\infty$ for all vertices $u$.

Examples. If $P$ is symmetric; that is, $\mathrm{p}(\mathrm{x}, \mathrm{y})=\mathrm{p}(\mathrm{y}, \mathrm{x})$ for all vertices $x$ and $y$, the walk is strongly reversible. Just set $m(u) \equiv 1$ and use the regularity of $P$. A walk is isotropic if $p(u, v)=1 /(q(u)+1)$ when $v$ is a neighbor of $u$. Recall that a regular walk is order bounded, so if we set $m(u)=1+q(u)$ we see that isotropic walks are strongly reversible.

One says that two functions $f$ and $g$ are equivalent and write $f \sim g$ if whenever either is non-zero so is the other and the ratios of their absolute values are bounded above and below by positive constants.

Lemma 20. If the walk $P$ is strongly reversible (regular and strongly transient) then $K_{T^{*}} \sim K_{S}$ and $K_{S^{*}} \sim K_{T}$.

Proof: Both results follow from showing that $K_{S}(u, v) \sim K_{T}(v, u)$. Referring to (11) and (12) in Section 4 we see that both expressions are zero if $v$ is not below $u$. If $u=v$ we are asking if $1-\alpha\left(u, u^{-}\right) \sim 1$ which follows since $1-\bar{\alpha} \leq 1-\alpha\left(u, u^{-}\right) \leq 1$. Thus we may assume that $v$ is below $u$ and that $u \neq v$. Referring again to (11) and (12) and using Theorem 3 we want to compare

$$
\frac{V(u, v)}{V(v, u)} \frac{A(v)}{A(u)} \alpha(v, u)
$$


to

$$
\alpha(v, u)-\alpha\left(v, u^{-}\right)=\alpha(v, u)\left(1-\alpha\left(u, u^{-}\right)\right) .
$$

We noted at the end of Section 3 that $A(u) \sim 1$ so we only need to show that

$$
\frac{V(u, v)}{V(v, u)} \sim 1
$$

for all vertices $u$ and $v$. Let us recall the definitions. Let $\omega=\left\{\omega_{0}, \omega_{1}, \ldots, \omega_{n}\right\}$ be the geodesic that connects $u=\omega_{0}$ to $v=\omega_{n}$. Then

$$
\frac{V(u, v)}{V(v, u)}=\frac{\prod_{j=1}^{n} p\left(\omega_{j-1}, \omega_{j}\right)}{\prod_{j=1}^{n} p\left(\omega_{j}, \omega_{j-1}\right)}
$$

Consider two special cases. If the walk is symmetric the expression in (21) is 1 and we are done. If the walk is isotropic it is easy to check that the expression in $(21)$ is

$$
\frac{q(u)+1}{q(v)+1}
$$

and since isotropic regular walks are order bounded we are done.

More generally, if the walk is reversible then

$$
\frac{p\left(\omega_{j-1}, \omega_{j}\right)}{p\left(\omega_{j}, \omega_{j-1}\right)}=\frac{m\left(\omega_{j-1}\right) p\left(\omega_{j-1}, \omega_{j}\right)}{m\left(\omega_{j}\right) p\left(\omega_{j}, \omega_{j-1}\right)} \frac{m\left(\omega_{j}\right)}{m\left(\omega_{j-1}\right)}=\frac{m\left(\omega_{j}\right)}{m\left(\omega_{j-1}\right)}
$$

and so the product in (21) is

$$
\prod_{j=1}^{n} \frac{m\left(\omega_{j}\right)}{m\left(\omega_{j-1}\right)}=\frac{m(u)}{m(v)} \sim 1
$$

since the walk is strongly reversible. This completes the proof.

Theorem 21. If $P$ is strongly reversible (regular and strongly transient) the the Green's operator is of weak type $(1,1)$, is bounded on $\ell^{p}, 1<p<\infty$, and satisfies the conclusion of Lemma 19 .

Proof: $S$ is bounded on $\ell^{p}, 1 \leq p<\infty$, and satisfies the conclusion of Lemma 13. $S^{*}$ is of weak type $(1,1)$ and is bounded on $\ell^{p}, 1<p \leq \infty$. Since $K_{T} \sim K_{S^{*}}, T$ is of weak type $(1,1)$, and is bounded on $\ell^{p}, 1<p \leq \infty$. $G=T S R$. $T, S$, and $R$ are bounded on $\ell^{p}, 1<p<\infty$ so $G$ is bounded on $\ell^{p}$. Fix $s>0$ and an $f \in \ell^{1}$.

$$
|\{x:|G f(x)|>s\}| \leq \frac{2}{\bar{\delta}}\|S R f\|_{1} \frac{1}{s} \leq C\|f\|_{1} \frac{1}{s} .
$$

Now take a finite set of vertices, $E$, and let $F$ be the subset described in Lemma 13. Then $|F|>\frac{1}{2}|E|$ and

$$
\left\|G \chi_{F}\right\|_{\infty} \leq\|R\|_{\infty}\left\|T\left(S \chi_{F}\right)\right\|_{\infty} \leq\|R\|_{\infty}\left\|S \chi_{F}\right\|_{\infty} \leq \frac{1}{\bar{\delta}}\|R\|_{\infty} .
$$


Theorem 22. $G=-\Delta^{-1}$ on $\ell^{p}, 0<p<\infty$.

Proof: Immediate from Theorem 4, Theorem 5, Theorem 8, and Corollary 12 .

Corollary 23. Under the conditions of Theorem 20, if $1<p<\infty$ maps $\ell^{p}$ onto itself.

6. Factorization of the Laplacian and Green's operator-The disk realization. We present here the modifications needed to carry over the results of Sections 4 and 5 . In this setting the paths start from a finite position, o instead of "coming from infinity". In this section we use the following notation: For $x \in \partial X, x=\left\{0=x_{0}, x_{1}, x_{2}, \ldots, x_{k}, \ldots\right\}$. Thus, $x_{k}$ is always a vertex at distance $k$ from 0 and if $k \geq 1$ then $\left(x_{k-1}\right)=\left(x_{k}\right)^{-}$. For $k \geq 1$ the $q\left(x_{k}\right) \equiv q$ lower neighbors of $x_{k}$ are denoted $x_{k+1, j}, j=1,2, \ldots, q$. For $x_{0}$ we have $q(0)+1 \equiv q+1$ lower neighbors $x_{1, j}, j=1,2, \ldots, q+1$.

We set,

$$
T f\left(x_{0}\right)=f\left(x_{0}\right)
$$

and if $n \neq 0$

$$
T f\left(x_{n}\right)=\sum_{k=0}^{n}\left(\alpha\left(x_{n}, x_{n-k}\right)-\alpha\left(x_{n}, x_{n-k-1}\right)\right) f\left(x_{n-k}\right)+\alpha\left(x_{n}, x_{0}\right) f\left(x_{0}\right) .
$$

For the "backward difference" we set

$$
\Delta^{-} f\left(x_{0}\right)=f\left(x_{0}\right)
$$

and if $n \neq 0$

$$
\Delta^{-} f\left(x_{n}\right)=\frac{1}{1-\alpha\left(x_{n}, x_{n-1}\right)} f\left(x_{n}\right)-\frac{\alpha\left(x_{n}, x_{n-1}\right)}{1-\alpha\left(x_{n}, x_{n-1}\right)} f\left(x_{n-1}\right) .
$$

It is easy to check that $T$ and $\Delta^{-}$are inverse to each other on the class of all functions on the tree. We see that $T$ is defined by the kernel $K_{T}$ wherc

$$
K_{T}(u, v)= \begin{cases}\alpha(u, v), & \text { if } v=0 \\ \alpha(u, v)-\alpha\left(u, v^{-}\right), & \text {if } u \text { is below } v \text { and } v \neq 0 . \\ 0, & \text { otherwise }\end{cases}
$$

$S$ is defined by the same formula as in Section 4; however, the values $\mu\left(I_{v}\right)$ are defined as in Section 3. Similarly,

$$
\Delta^{+} f\left(x_{n}\right)=\sum_{j} \pi\left(x_{n}, x_{n+1, j}\right) f\left(x_{n+1, j}\right)-f\left(x_{n}\right) .
$$

With $A$ defined as in Section 3 we find that

$$
\Delta^{+} \Delta^{-} f(x)=R(x) \Delta f(x) .
$$

We find again that $G=T S R$ and the results of Sections 4 and 5 carry over almost without change. 
7. Examples. The simplest example is an isotropic walk on a homogeneous tree of degree $q+1, q \geq 2$, in the half-plane realization. By symmetry we see that $\pi\left(u^{-}, u\right) \equiv \pi=1 / q$ for all vertices $u$. Similarly, $\alpha \equiv \alpha\left(u, u^{-}\right)$for all $u$. Using equation (4) we have

$$
\alpha=\frac{1}{q+1}+\frac{q}{q+1} \alpha^{2}
$$

from which it follows that $\alpha=1 / q$. From equation (5) we see that $A(u)=(q-1) /(q+1)$ for all vertices $u$. We also see that except for a factor of $(q-1) / q$ the operators $S$ and $T$ are adjoints. The Green's kernel is given by

$$
G(u, v)=\frac{q}{q+1}\left(\frac{1}{q}\right)^{d(u, v)}
$$

which follows by a trival application of Theorem 11. Since $\left(G \chi_{\left\{x_{0}\right\}}\right)(x)=$ $G\left(u, x_{0}\right)$ we see that the Green's operator is not bounded on $\ell^{\prime}$.

An example that arises from a group is a homogeneous tree with a symmetric anisotropic walk. For a specific example take a group with identity $e$ and three generators $a, b$, and $c$; with the relations $a^{2}=b^{2}=c^{2}=c$. Let $Y$ be the Cayley graph of the group. It is a tree that is homogeneous of order three. Each vertex corresponds to a reduced word and the three edges at each vertex correspond to the right multiplication by one of the three generators. Assign to each edge $p(g), g=a, b$, or $c$, as the case may be, where $p(a)+p(b)+p(c)=1$, and each of the $p$ 's is positivc. For any such assignment of probabilities the associated walk is regular, strongly transient, and strongly reversible.

Let us now construct a simple example for which the Green's operator fails to be bounded on $\ell^{p}, 1<p<\infty$. Take a tree that is homogeneous of order three in its half-plane realization. At each vertex there is onc neighbor directly above it and two directly below. To the edge going up assign the probability 4 and to the two edges going down split the difference and give earh the probability 3 . What makes this example work is that $1 / 3<0.4<1 / 2$. If the "up" probability is grcater than or equal to $1 / 2$ the walk fails to be transient and if it is less than $1 / 3$ the Green's operator is bounded on $l^{1}$. The case where it is equal to $1 / 3$ gives the isotropic walk. Take the probabilities as we gave them. From equation (4) we have the equation

$$
\alpha=.4+.6 \alpha^{2}
$$

where $\alpha$ is the visiting probability associated with an upward transition. From this equation we see that $\alpha$ is equal to $2 / 3$. The argument of Theorem 19 shows that $T$, and so also $G$, is bounded on $\ell^{p}$ if $p>\log 3 / \log (3 / 2)=p_{0}$, which is about 2.7. Testing $T$ against the characteristic function of a vertex we find that the result is sharp and that $T$ fails to be bounded on $\ell^{p}$ if $p \leq p_{0}$. A more careful analysis shows that $T$ is of restricted weak type $\left(p_{0}, p_{0}\right)$. (See [SW; p.197] for definitons.) 
8. Comments and questions. The principal boundedness results in this paper follow from an isoperimetric inequality expressed in Lemma 13, which then leads to the weak-type estimate stated in Theosem 14. This circle of ideas is closely related to the results obtained by Gerl in [G], where he obtains $\ell^{p}$ boundedness results starting from another isoperimetric inequality. While the lower boundary described in the proof of Lemma 13 is not the same as the boundary used by Gerl (and others in combinatoric graph theory) there must be close connections and those connections should be studied. The notion of a lower boundary was introduced in [RT] in a different setting. The possibility that it could lead to boundedness results for the Green's operator came to mind when we learned of Gerl's work in [G].

In [RT] Lemma 13 in the setting of the dyadic martingale was used to compute the $K$-functional for the spaces $C M_{d}$, the space of discrete Carleson Measures, and $\ell^{0}$, the space of functions with finite support. In this special case the dyadic martingale is the boundary martingale of an isotropic random walk on a tree that is homogeneous of degree three; the nodes of the graph can be viewed as the index set for the dyadic intervals of $\mathbf{R}$. Lemma 13 allows the extension of the interpolation results to the boundary martingale of any strongly transient random walk on a non-homogeneous tree. In this more general setting the nodes of the tree represent intervals in a nested system of intervals more general than the dyadic system.

In Theorem 19 we find a $p_{0}$ such that the Green's operator is bounded on $\ell^{p}$ if $p>p_{0}$. This $p_{0}$ depends on $\bar{q}+1$, the maximum order of a vertex, and $\bar{\alpha}$, the "maxirnum visiting probability", $\bar{\alpha}=\sup a\left(u, u^{-}\right)$. For isotropic random walks on a homogeneous tree of order $q+1, q$ and $\alpha$ are constants, $\alpha=1 / q$, and this leads to $p_{0}=1$. For isotropic random walks on an order bounded tree we know that $p_{0}=1$ but the argument of Theorem 19 leads to a gross overestimate of $p_{0}$. In [L] Lyons uses the notion of an average branching number for a random walk on a tree where the branching number at a vertex $u$ is $q(u)$ in our notation. Lyons shows in a variety of problems that this average branching number behaves like $q$ when $q$ is a constant. Lyons' results suggest that there might be an "average visting probability" as well as an average branching number and that the infimum of those values of $p$ for which the Green's operator is bounded on $\ell^{p}$ could be computed from these averages.

Consider now the situation when the randon walk is strongly transient and strongly reversible. In this situation the Green's operator maps $\ell^{p}$ onto $\ell^{p}$ if $1<p<\infty$ and it is never bounded on $\ell^{1}$. For if it were bounded on $\ell^{1}$ then $T$ would be bounded on $\ell^{1}$, which implies that $S$ is bounded on $\ell^{\infty}$. But $S$ is never bounded on $\ell^{\infty}$ as we saw in our remarks following the proof of Lemma 6 . This raises the problem of describing the class of integrable functions, $f$, on the tree such that $G f$ is also integrable. We can define a kind of Hardy space, $H=\left\{f: f \in \ell^{1}, G f \in \ell^{1}\right\},\|f\|_{H}=\|f\|_{1}+\|G f\|_{1}$. It is easy to see that this norm is equivalent to an "atomic norm" in the sense that there are functions called atoms and $f \in H$ if and only if $f=\sum \lambda_{k} a_{k}(x)$ where the $a_{k}$ are atoms 
and $\sum\left|\lambda_{k}\right|<\infty$ where $\|f\|_{H} \sim \inf \sum\left|\lambda_{k}\right|$ over all such representations. This is not very satisfactory since the definition of "atom" is restrictive (an atom being the Laplacian of a point mass). One would want a less restrictive definition of an atom as well as a maximal function characterization before one would have a satisfying theory. This line of thought also suggests that there should be a $B M O$ theory. We only remark that the tree as a measure space with discrete measure and the natural metric induced by geodesic distance is not a space of homogeneous type, in the sense of Coifman and Weiss [CW]. For spaces of homogeneous type Coifman and Weiss constructed a Hardy space theory.

\title{
References
}

[C] P. Cartier, Fonctions harmonique sur un arbre, Symp. Math. 9(1972), 203-270.

[CW] R.R. CoIfMan and G. WeIss, Extensions of Hardy spaces and their use in Analysis, Bull. Amer. Math. Soc. 83 (1977), 569-645.

[G] P. GERL, Random walks on graphs with a strong isoperimetric inequality, J. Theoretical Prob. 1 (1988), 171-188.

[KPT] A. Korányi, M. Picardello, and M. Taibleson, Hardy spaces on non-homogeneous trees, Symp. Math. 29 (1988), 205-265.

[L] R. LyONS, Random walks and percolation on trees, Ann. Prob. (to appear).

[RT] R. ROCHBERG AND M. TAIBLESON, An averaging operator on a tree, in "Harmonic Analysis and Partial Differential Equations," Lectures Notes in Math. 1384, Springer Verlag, 1989, pp. 207-213.

[RW] R. ROCHBerg AND G. WeIss, Derivatives of analytic families of Banach spaces, Annals Math. 118 (1983), 315-347.

[SW] E.M. STEIN AND G. Weiss, "Introduction to Fourier Analysis on Euclidean Spaces," Princeton Univ. Press, 1971.

\author{
Department of Mathematics \\ Campus Box 1146 \\ Washington University \\ Saint Louis, Missouri 63130 \\ U.S.A. \\ rr@david.wustl.edu
}

